\title{
Preparation of Monodisperse Giant Unilamellar Anchored Vesicles Using Micropatterned Hydrogel Substrates
}

\author{
Jennifer Schultze, ${ }^{\S}$ Apostolos Vagias, ${ }^{*}, \|$ Lijun Ye, ${ }^{\S}$ Ephraim Prantl, ${ }^{\S}$ Valentina Breising, ${ }^{\S}$ Andreas Best, ${ }^{\S}$ \\ Kaloian Koynov, ${ }^{*}, \S$ Carlos M. Marques, ${ }^{*},{ }^{\circledR}$ and Hans-Jürgen Butt ${ }^{\S \odot}$ \\ ${ }^{\S}$ Max Planck Institute for Polymer Research, Ackermannweg 10, 55128 Mainz, Germany \\ "Zernike Institute for Advanced Materials, Macromolecular Chemistry and New Polymeric Materials, University of Groningen, \\ Nijenborgh 4, NL-9747 AG Groningen, Netherlands \\ \#Institut Charles Sadron, Université de Strasbourg, CNRS, 23 rue du Loess, 67034 Strasbourg, France
}

Supporting Information

\begin{abstract}
Giant unilamellar vesicles (GUVs) are model membrane systems consisting of a single lipid bilayer separating an inner lumen from the outer solution, with dimensions comparable to that of eukaryotic cells. The importance of these biomimetic systems has recently grown with the development of easy and safe methods to assemble GUVs from complex biorelevant compositions. However, size and position control is still a key

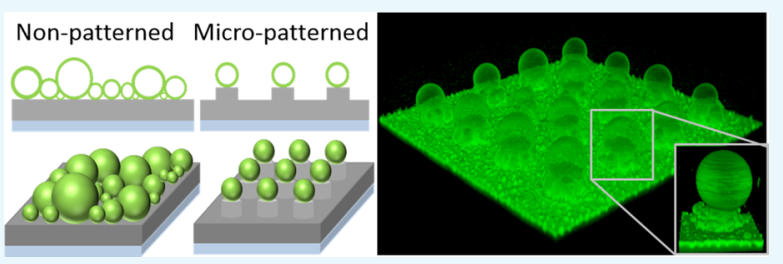
challenge for GUV formation and manipulation. Here, a gelassisted formation method is introduced, able to produce arrays of giant unilamellar anchored vesicles (GUAVs) with a predetermined narrow size distribution. The approach based on micropatterned gel substrates of cross-linked poly $(N$ isopropylacrylamide) allows performing parallel measurements on thousands of immobile unilamellar vesicles. Such power and flexibility will respond to the growing need for developing platforms of biomimetic constructs from cell-sized single bilayers.
\end{abstract}

\section{INTRODUCTION}

Model lipid membranes are essential tools for unraveling the mechanisms that drive complex biological processes at the level of cell membranes. ${ }^{1,2}$ In the family of available model lipid platforms, giant unilamellar vesicles (GUVs) strike as a prominent member. ${ }^{3}$ These cell-sized vesicles are composed of a single bilayer that separates an enclosed volume from the outer solution. Giant vesicles were first used to study the intrinsic physical and chemical properties of membranes: permeability, lipid mobility, mechanical behavior, and phase transformations. ${ }^{4,5}$ Today, they are employed not only in biophysics and biology but also in physical chemistry in quests for understanding the origin of life and in several applicationdriven fields such as microreactor synthesis or drug delivery. ${ }^{6-8}$

The first methods available for the formation of GUVs were often limited to a restricted variety of lipids. Over the past decade, many new strategies were developed for selfassembling giant vesicles from increasingly complex compositions of lipids and other (bio)macromolecules. ${ }^{8}$ The concomitant development of new methods for their observation and micromanipulation has now brought giant vesicles to the central stage of studies related to lipid bilayers. Here, the widening community of users calls for accuracy and predictability in the size distribution, for simplification of the methods for position control and for progress in the statistical power of the analysis performed on individual GUVs.

In this paper, we address these challenges by combining the advantages of gel-assisted methods for GUV formation with the precision and flexibility offered by gel micropatterning. Although photolithography and microcontact printing techniques have been previously used to attempt size control of GUVs, these attempts lacked the advantages of gel-growing methods and did not achieve good vesicle unilamellarity by gentle hydration or required electrical fields to induce unilamellar vesicle formation. $^{9-15}$ Photolithographic masks $^{-1}$ on an agarose gel circumvented some of these difficulties but controlled only the spatial distribution of spread lipids, leading to vesicle size polydispersity above $30 \% .{ }^{16}$ Recently, giant lipid vesicles were prepared also on $\mathrm{PDMS}^{17}$ and $\mathrm{Si}^{18}$ microwell arrays. As we will show here by appropriate patterning and cross-linking of the gel films, one can generate arrays of thousands of monodisperse giant unilamellar vesicles anchored on the substrate. We coin this new platform as giant unilamellar anchored vesicles (GUAVs) and demonstrate its advantages by performing fluorescence correlation spectroscopy (FCS) experiments on the immobilized vesicles and parallel measurements of membrane area changes induced by oxidative stress.

\section{RESULTS AND DISCUSSION}

2.1. Preparation of Micropatterned Hydrogel Layers. Our strategy for preparation of micropatterned, anchored

Received: April 1, 2019

Accepted: May 16, 2019

Published: May 29, 2019 

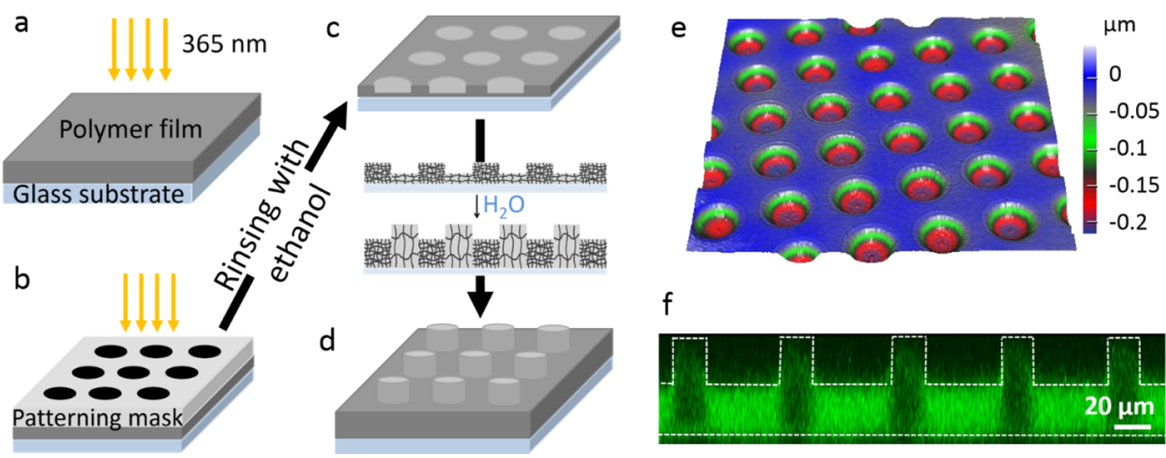

Figure 1. Preparation of micropatterned PNIPAAm hydrogels. (a) A PNIPAAm terpolymer film is spin-coated on a functionalized glass substrate and illuminated with a weak dose $\left(0.28 \mathrm{~J} / \mathrm{cm}^{2}\right)$ of collimated UV light $(365 \mathrm{~nm})$ to obtain an anchored, cross-linked polymer network. (b) Further UV illumination $\left(3.92 \mathrm{~J} / \mathrm{cm}^{2}\right)$ through a photolithography mask and $(c)$ subsequent rinsing with ethanol leads to the formation of a PNIPAAm film with a defined pattern of shallow, weakly cross-linked areas. (d) Submersion in aqueous solutions creates a PNIPAAm hydrogel in which the weakly cross-linked areas swell more than the rest of the film and form a pillar pattern. (e) White-light confocal profilometry image of a dry structured PNIPAAm film. (f) Confocal laser scanning microscopy image ( $x z$ view) of the patterned PNIPAAm hydrogel, swollen in phosphate buffered saline (PBS) containing a small amount of rhodamine 6G that adsorbs to the gel (see text for details).

hydrogel layers is schematically shown in Figure 1. As a hydrogel-forming material, we used a poly $(N$-isopropylacrylamide)-based statistical terpolymer (PNIPAAm). Its backbone included $94 \%$ of NIPAAm units as a main component, approximately $5 \%$ of ionizable methacrylic acid units for increased solubility in water, and $1 \%$ hydrophobic benzophenone-based cross-linker (see the Supporting Information for the reaction).

The procedures for the synthesis of the PNIPAAm terpolymer and its processing into thin anchored hydrogel layers are well established in our laboratory and described in detail in the Methods. ${ }^{19-22}$ Briefly, a thin PNIPAAm film (1.1 $\pm 0.1 \mu \mathrm{m}$ in the dry state) was spin-coated from an ethanol solution on glass substrates prefunctionalized with benzophenone silane. The film was illuminated with collimated UV light (Figure 1b) that lead to activation of the benzophenone groups on both the polymer chains and the glass surface and therefore to simultaneous cross-linking and anchoring of the PNIPAAm network. ${ }^{20,23}$ By tuning the exposure dose of the UV light, one can control the degree of cross-linking and thus the swelling ratio $R_{S}$ of the PNIPAAm hydrogel films (see Figure $S 1$ and Table S1 for details). To prepare micropatterned hydrogels, an initial homogeneous UV illumination of the PNIPAAm film at a low-energy dose (Figure 1a) that ensured anchoring and lowdensity cross-linking of the entire film was followed by further UV illumination through a photolithography mask (Figure 1b). The mask covered a square pattern of circles on the PNIPAAm film and protected them from further illumination and crosslinking. The noncovered parts were illuminated further, leading to the formation of a strongly cross-linked background area on the film. After the illumination, the PNIPAAm film was rinsed with ethanol, thus removing the non-cross-linked and nonanchored polymer chains. This resulted in the formation of a dry polymer film with shallow circular holes with defined diameters ordered in a precise square pattern (Figure 1c). The polymer network in these holes is less cross-linked than its surroundings, and upon swelling with water, it swells more creating a pattern of strongly swollen hydrogel pillars on a less swollen background (Figure 1d). A typical white-light confocal profilometry image of a dry patterned PNIPAAm film after rinsing with ethanol is shown in Figure 1e. The square pattern of round holes with a depth of $0.3 \mu \mathrm{m}$ is clearly visible. In these weakly illumined regions, more polymer chains were not cross- linked and rinsed off by the ethanol. The rest of the film itself has a thickness of $1 \mu \mathrm{m}$. Below the holes, a $0.7 \mu \mathrm{m}$ thick layer of polymer remains. Figure if shows a confocal laser scanning microscopy (CLSM) image ( $x z$ view) of the same patterned PNIPAAm film after swelling in phosphate buffered saline (PBS). To enable CLSM imaging, a small amount of fluorescent rhodamine $6 \mathrm{G}$ molecules that preferably adsorb to the PNIPAAm due to hydrophobic interactions ${ }^{22}$ was added to PBS. The image reveals a pattern of strongly swollen $\left(R_{\mathrm{S}} \approx\right.$ $30)$ hydrogel pillars on top of the less swollen $\left(R_{\mathrm{S}} \approx 10\right)$ background.

2.2. Formation of Monodisperse Giant Unilamellar Anchored Vesicles. GUAVs were prepared by hydration of a dry layer of lipids spread on the surface of a dry patterned PNIPPAm film (Figure 2). As a model membrane system, we used 1,2-dioleoyl-sn-glycero-3-phosphocholine (DOPC) and 1,2-dioleoyl-sn-glycero-3-phosphoserine (DOPS) in a 4:1 ratio. A small amount (0.5\%) of Atto488-labeled 1,2-dioleoyl-snglycero-3-phosphoethanolamine (Atto488-DOPE) was added to enable confocal microscopy imaging. First, a lipid layer was deposited on top of a dry patterned PNIPAAm film by drop casting $30 \mu \mathrm{L}$ of chloroform solution $(1 \mathrm{~g} / \mathrm{L})$. The lipid solution spread over the entire surface of the patterned PNIPAAm film and the chloroform evaporated within minutes under ambient conditions. Afterward, the microscope-coverslip glass substrate supporting the PNIPAAm film was mounted on an Attofluor Cell Chamber (Thermo Fisher Scientific) with the lipid layer facing upward, and $600 \mu \mathrm{L}$ of phosphate-buffered saline (PBS) was slowly added to the chamber. Within $5 \mathrm{~min}$, the background gel swelled to a thickness of approximately 10 $\mu \mathrm{m}$ (that corresponds to $\left.R_{\mathrm{S}} \approx 10\right)$ and the pillars to $20 \mu \mathrm{m}\left(R_{\mathrm{S}}\right.$ $\approx 30$ ), and numerous giant vesicles formed. The experiments were performed at room temperature, $23{ }^{\circ} \mathrm{C}$.

Vesicle formation speed and mechanisms are comparable to that observed on PVA gels or agarose substrates. ${ }^{24,25}$ However, in contrast to these early works that used homogeneous substrates and thus the GUVs form randomly and have a very broad size distribution (Figure 2a), on the micropatterned PNIPAAm film, giant vesicles can form only on the weakly cross-linked and strongly swollen pillars (Figure 2b). Furthermore, the giant vesicles have a well-defined size determined by the pillars' diameter (Figure 2d). On the strongly cross-linked parts of the film, vesicle formation is 

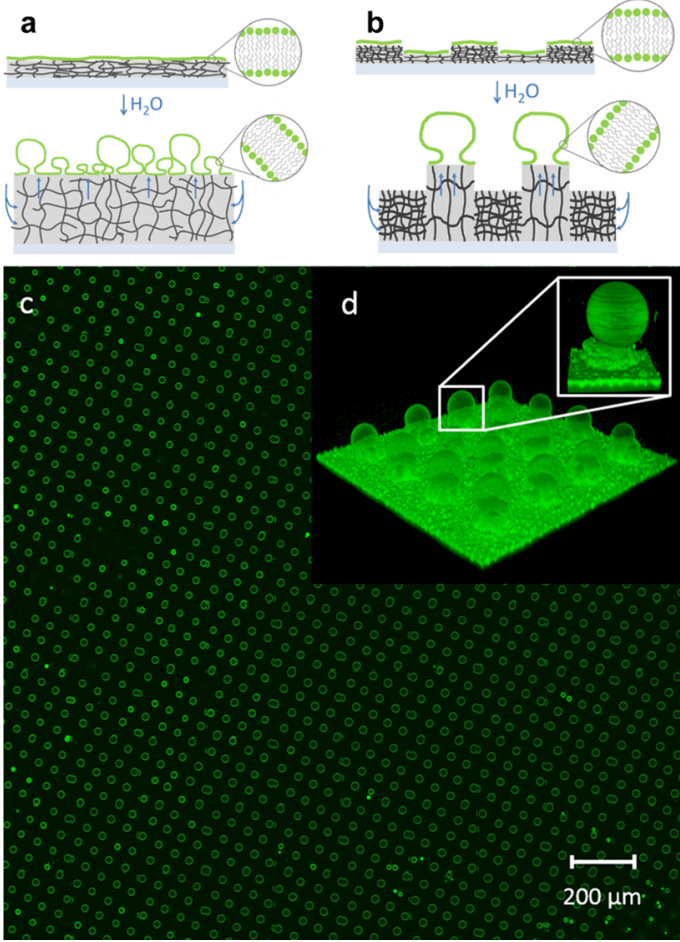

Figure 2. Formation of giant vesicles. Schemes of GUVs forming (a) with various sizes on a homogenous hydrogel film and (b) with a defined size on the pillars of a micropatterned hydrogel film. A dry layer of lipids spread on the surface of a dry PNIPAAm gel is hydrated from water coming from below (blue arrows) and swelling the gel. (c) 2D CLSM image of an array of uniform GUAVs, prepared on a micropatterned PNIPAAm hydrogel. (d) 3D CLSM image of an array of 16 GUAVs zoomed in to a single pillar with one GUAV on top.

inhibited (see Figure S2 and the Supporting Information for details), and only very small vesicles grew in these areas. The 3D CLSM images in Figure 2d reveal that the lipids not only form vesicles on the top of the PNIPAAm hydrogel pillars but also partially penetrate into the polymer network. Thus, the unilamellar vesicles are immobilized on controlled positions, according to a predefined pattern that is a promising alternative to other immobilization methods. ${ }^{26,27}$ We name this new type of lipid vesicle system Giant Unilamellar Anchored Vesicles (GUAVs). Thousands of such uniformly sized GUAVs ordered in a predefined pattern (Figure 2c) can be prepared in a few minutes. Similar to the vesicles formed on PVA gels or agarose substrates, ${ }^{24,25}$ the anchored vesicles prepared on the micropatterned PNIPAM hydrogels are unilamellar as indicated by the similar fluorescence brightness that they show on the CLSM images. The fact that in Figure 2c some smaller vesicles appear brighter than the rest is caused by the limited resolution of the shown image as demonstrated in Figure S3.

Furthermore, the size of the GUAVs can be easily controlled by tuning the diameter of micropatterned PNIPAAm pillars. This is demonstrated in Figure 3 that shows CLSM images of GUAVs formed on PNIPAAm films patterned with three different photolithography masks. The diameters of the nontransparent circles were 15,30 , and $50 \mu \mathrm{m}$. With diameters of $15.8 \pm 3.1,30.9 \pm 2.4$, and $46.1 \pm 2.8 \mu \mathrm{m}$, the generated GUAVs closely match the sizes defined by the photolithography masks. Note that such a close correspondence of sizes cannot be determined only by the amount of lipids

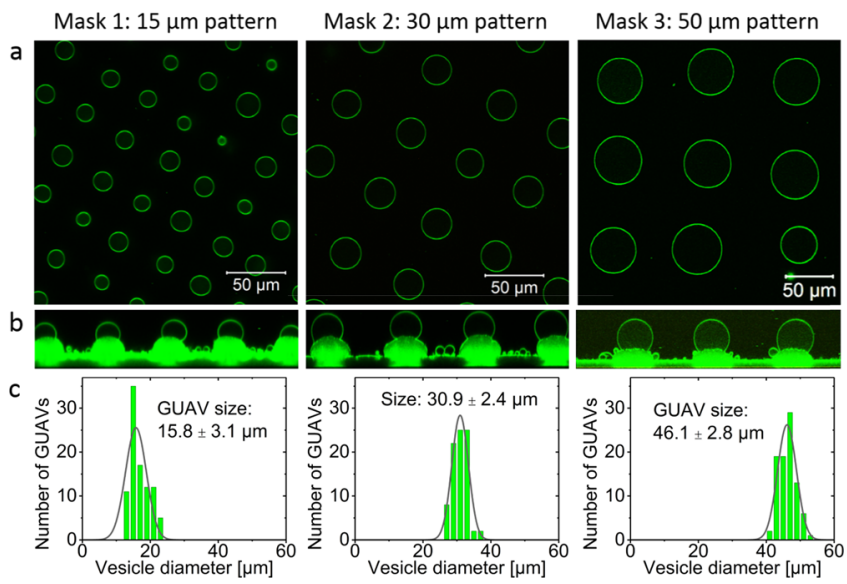

Figure 3. Controlling the vesicle size. GUAVs prepared by PNIPAAm gel-assisted swelling for three different mask/pattern sizes. (a) CLSM images ( $x y$ plane) of GUAVs, labeled with Atto488. (b) CLSM images ( $x z$ plane) of GUAVs. (c) Size distribution of the GUAV diameters. The histograms were obtained by measuring the diameters of roughly 90 GUAVs for each pattern. The lines represent a normal distribution fit that yielded the values of the average GUAV diameter and the respective standard deviation.

available on the pillar before swelling since there is a factor of four between the surface of a sphere and the surface of its twodimensional projection; formation of a vesicle from a bilayer patch of radius $R$ should result in a vesicle of radius $R / 2$. For the GUAV technique, it is likely that the anchoring conditions of the bilayer on the pillar, as seen in Figures $2 \mathrm{~d}$ and $3 \mathrm{~b}$, also play an important role in controlling the vesicle size.

The strategy developed in this paper relies on the differential swelling ratios between gel regions of high and low crosslinking densities. After swelling, the highly swollen regions of the gel appeared as pillars decorated with a giant vesicle. The hard regions surrounding the pillars did not lead to giant vesicle growth. Thus, the parameters of the pattern of the optical mask controlling the geometry of the high and low cross-linked regions translated directly into giant vesicle size and positioning. The method will allow preparing not only giant vesicles with monodisperse size but also more complex but well-defined mixtures of vesicles with different sizes anchored on predetermined positions. In particular, it could be useful to prepare arrays of GUAVs with sizes gradually changing, for example, between $50 \mu \mathrm{m}$ and $5 \mu \mathrm{m}$, and study the effect of vesicle size in a single microscopy experiment.

Such an approach takes advantage of the anchoring of GUVs after the swelling of the hydrogel film. While the detailed nature of this anchoring is not completely clear yet, most likely, it originates from hydrophobic attraction between the lipids and the hydrogel films. As discussed above, as the hydrogelforming material, we used a statistical terpolymer (PNIPAAm) which backbone contains also $1 \%$ of hydrophobic benzophenone-based cross-linker units. In previous studies, we have found that the presence of these units leads to hydrophobic attraction between similar PNIPAAm hydrogels and partially hydrophobic molecules such as immunoglobulin ${ }^{28}$ or rhodamine $6 \mathrm{G} .{ }^{22}$ The existence of such attraction is also indicated by the fact that a large number of lipids are partitioned inside the swollen hydrogels as shown in Figure $3 \mathrm{~b}$. Furthermore, electrostatic interactions can also be present and eventually lead to systematic differences in the partitioning behavior of charged and uncharged lipids. 


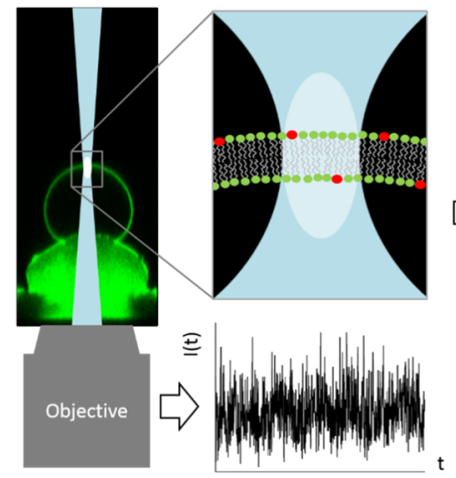

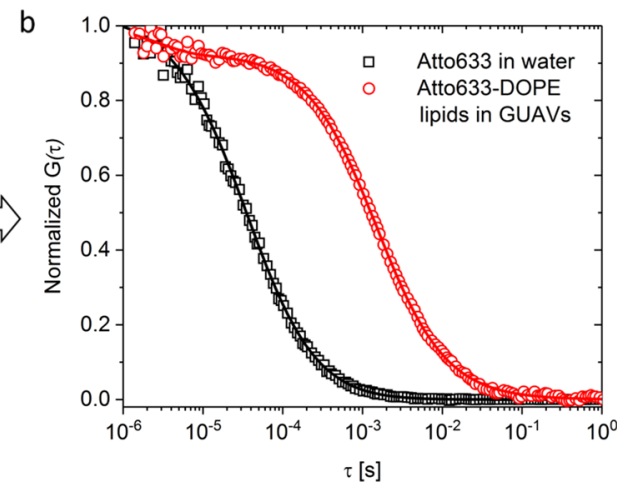

Figure 4. Lipid diffusion in the bilayer membrane of a GUAV measured by fluorescence correlation spectroscopy. (a) Schematic of the experiment. The fluorescence (green) of the Atto488-DOPE lipids present in the GUAV membrane at a $0.5 \%$ fraction is used to image the vesicles and position the confocal volume precisely at the lipid membrane. Next, excitation at $633 \mathrm{~nm}$ is used to excite the Atto633-DOPE lipids that are present at a low $(0.01 \%)$ fraction suitable for FCS experiments. The temporal fluctuations of the detected fluorescence intensity $I(t)$ caused by the lateral diffusion of the Atto633-DOPE lipids (red) through the confocal volume are recorded and processed to an autocorrelation curve. (b) Normalized autocorrelation curve $G(\tau)$ measured for Atto633-labeled DOPE lipids diffusing in the bilayer membrane (red circles) and the corresponding fit (red solid lines) with a 2D diffusion model function. The autocorrelation curve measured for the reference dye used for calibration of the confocal volume, Atto633, freely diffusing in water (black squares) and the corresponding fit (black solid lines) with a 3D diffusion model function are also shown for comparison.

2.3. Lipid Mobility in the Bilayer Membrane of GUAVs. To demonstrate the advantages of anchored giant vesicles with respect to position control, we investigated the mobility of lipids by fluorescence correlation spectroscopy (FCS) ${ }^{29,30}$ Due to its high selectivity, sensitivity (down to a single-molecule level), and extremely small probing volume $\left(<1 \mu \mathrm{m}^{3}\right)$ that can be precisely positioned on the lipid membrane, FCS is uniquely suited for such studies. ${ }^{29,30}$ Here, we study the lateral diffusion of Atto633-DOPE (Atto633-1,2dioleoyl-sn-glycero-3-phosphoethanolamine) lipids in the membrane of the GUAVs (Figure 4). Therefore, $0.01 \%$ of Atto633-DOPE was added to the standard lipid mixture used for the preparation of GUAVs. The GUAVs were first imaged with CLSM at $488 \mathrm{~nm}$, the excitation wavelength of Atto488DOPE lipids present in the membrane at the $0.5 \%$ fraction. Next, the confocal volume was positioned precisely at the lipid membrane on the "north pole" of a GUAV (Figure 4a), and FCS measurements were performed with the Atto633-DOPE lipids using an excitation wavelength of $633 \mathrm{~nm}$. Due to the anchoring of the GUAVs, the lipid membrane position was stable with respect to the confocal volume, and thus FCS experiments could be performed over many minutes.

A typical FCS autocorrelation curve measured in this way is shown in Figure $4 \mathrm{~b}$ together with the corresponding fit with an analytical function based on a single-component two-dimensional diffusion model. ${ }^{29,30}$ The fit yielded a value of $D=(1.01$ $\pm 0.14) \times 10^{-11} \mathrm{~m}^{2} / \mathrm{s}$ for the diffusion coefficient of Atto633DOPE lipids. This value is in good agreement with the results of earlier FCS studies of labeled lipid diffusion in lipid GUVs. ${ }^{31-33}$

2.4. Photo-Oxidation of the GUAV Membrane. The GUAV platform allows following simultaneously the physicochemical transformation of lipid bilayers on many vesicles. As an example, we studied lipid photosensitization. ${ }^{34-36}$ Photosensitizer erythrosine $(50 \mu \mathrm{M})$ was added to phosphate buffered saline (PBS) and irradiated at $547 \mathrm{~nm}$ to create singlet oxygen that oxidizes the lipid bilayers. ${ }^{34,35}$ Under such conditions, membrane area changes are expected following the formation of hydroperoxides, the primary product of photosensitization. $^{37-39}$ Therefore, we monitored the area increase of the GUAVs equator as a function of the irradiation dose. For these particular experiments, an array of $9 \times 9$ GUAVs was chosen (Figure 5), but much larger numbers of
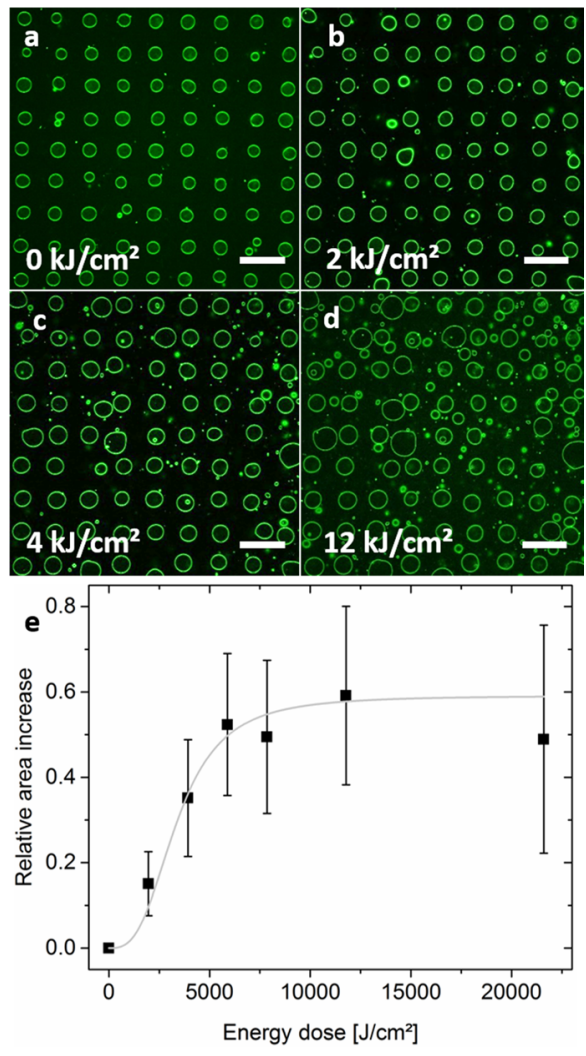

Figure 5. Photo-oxidation experiments on GUAVs. Confocal laser scanning images of the equator of GUAVs after illumination with different irradiation doses. (a) Before irradiation the GUAVs are uniform and completely round-shaped. With irradiating the photooxidizer erythrosine, the GUAVs become larger, deform, and move from their positions. (b-d) The effect increases with increasing dose. Scale bars are $50 \mu \mathrm{m}$. (e) Area increase of the GUAV equator as a function of energy dose. 
vesicles can be monitored depending on the target resolution. As the images show, the fate of each individual vesicle in the array can be easily monitored, and measurements such as the changes in the relative area of the equatorial plane (Figure 5e) can be straightforwardly performed by image analysis.

Variations in values of the area increase are intrinsic to this process and also seen in GUVs, as for instance reported in ref 38 , although they are likely amplified here by heterogeneities in the anchoring conditions between the membrane and the gel.

\section{CONCLUSIONS}

We have shown here that the ability to spatially modulate the crosslinking density of a thin gel brings unprecedented size control in the gel-assisted formation of single-bilayer vesicles. By patterning the cross-linking density in thin gels of PNIPAAm, we have obtained essentially monodisperse giant anchored vesicles with a variation in diameters smaller than $10 \%$.

The strategy developed in this paper relied on the differential swelling ratios between gel regions of high and low cross-linking densities. After swelling, the highly swollen regions of the gel appeared as pillars decorated with a giant vesicle, the hard regions surrounding the pillars did not lead to giant vesicle growth. This happened because the vesicle formation process is strongly dependent on the degree of cross-linking and the swelling ratio even for unstructured PNIPAAm gels (see Figure S2 and the Supporting Information for details). While a large number of vesicles with various sizes form on such gels, there is a general tendency that the average size and the fraction of large vesicles are decreasing gradually with the increase of cross-linking density (Figure S2). Thus, the parameters of the pattern of the optical mask controlling the geometry of the high and low cross-linked regions translated directly into giant vesicle size and positioning. The transferability from pattern design to giant vesicle properties is an intrinsic flexible powerful feature of the method, as it will allow preparing not only giant vesicles of a targeted size but also more complex but well-defined mixtures of different sizes with predetermined positions.

Indeed, contrary to gel-assisted growth with other polymer gels, the giant vesicles here remained attached to the substrate and positioned on the pillars. As a result, thousands of anchored, precisely positioned giant vesicles are formed upon simple addition of the solution to the dry patterned gel where lipid was spread. The anchoring is due to the lipid penetration into the dry and swollen gel, which changes the capillary boundary conditions between the bilayers and the substrate. Although the detailed structure of the anchoring region is likely to determine how well the interior of the GUVs is sealed with respect to different molecules, it does not compromise the purity of the formed GUAVs since the gel is chemically crosslinked. It is important to underline that the stability of this giant vesicle platform is insured also by the chemical crosslinking of the gel film to the substrate, resulting in a very robust formation and observation platform for GUAVs.

Physical measurements performed on GUAVs revealed also the possibilities offered by this platform with respect to position control and parallel measurements under a microscope. FCS measurements could be performed under very stable conditions for several minutes, providing lipid diffusion coefficients in agreement with values from the literature. Photosensitization of the vesicles was also easily achieved, and the resulting transformations could simultaneously be followed and qualitatively evaluated on many vesicles. Excitingly, these achievements are a key step toward the development in a near future of automated measurements under an optical microscope on massive arrays of biomimetic lipid membranes.

\section{MATERIALS AND METHODS}

4.1. 4-Methacryloyloxybenzophenone (MABP). Ten grams $(50 \mathrm{mmol})$ of 4-hydroxybenzophenone was dissolved in $100 \mathrm{~mL}$ of dichloromethane (DCM), the mixture was cooled in an ice bath, and a solution of $5.1 \mathrm{~mL}(53 \mathrm{mmol})$ of methacryloyl chloride and $7.7 \mathrm{~mL}(56 \mathrm{mmol})$ of triethylamine in $20 \mathrm{~mL}$ of dry DCM was added dropwise. The reaction was stirred at room temperature for $4 \mathrm{~h}$. The solvent was evaporated, and the residue was dissolved in diethyl ether. The nonsoluble triethylammonium salt was removed by filtration. The organic phase was washed with water three times and afterward dried over $\mathrm{Na}_{2} \mathrm{SO}_{4}$. The solvent was evaporated, and the crude product was chromatographed over $\mathrm{DCM} /$ hexane $(7: 3)$. The product was recrystallized from a mixture of DCM and hexane (2:8).

4.2. Functionalization of the Glass Substrates. Round microscope cover slides (diameter: $25 \mathrm{~mm}$, thickness: $150 \mu \mathrm{m}$ ) were used as substrates for the polymer films. The glass slides were cleaned with $2 \%$ Hellmanex solution (Hellmanex II, Hellma $\mathrm{GmbH}$, Müllheim), ultrapure water (Milli-Q water, 18.2 $\mathrm{M} \Omega \mathrm{cm}$ ), and twice with ethanol (each time with $15 \mathrm{~min}$ sonication in the corresponding solvent/solution). Afterward, the glass slides were stored for at least $24 \mathrm{~h}$ in $6 \%$ benzophenone triethoxysilane ethanol solution under argon in the dark. Then, the slides were washed with ethanol and dried under vacuum for $1 \mathrm{~h}$ at $50{ }^{\circ} \mathrm{C}$.

4.3. Poly(N-Isopropylacrylamide)-Based Terpolymer. Ten grams ( $88 \mathrm{mmol})$ of $N$-isopropylacrylamide (NIPAAm) (recrystallized from toluene/hexane, 1:1), $380 \mathrm{mg}(0.37 \mathrm{~mL}$, $4.4 \mathrm{mmol}$ ) methacrylic acid (MAA) (distillated before use), and $235 \mathrm{mg}(0.88 \mathrm{mmol})$ 4-methacryloyloxybenzophenone (MABP) were dissolved in $68 \mathrm{~mL}$ dioxane. Argon was flown through the solution for $1 \mathrm{~h}$. Sixty-seven milligrams $(0.41$ $\mathrm{mmol}$ ) of azobisiosbutyronitrile (AIBN) was added to the solution, and the reaction was stirred at $60{ }^{\circ} \mathrm{C}$ under argon for $48 \mathrm{~h}$. For purification, the reaction solvent was evaporated, and the product precipitated from methanol in ice cold diethyl ether. The molecular weight was $M_{\mathrm{w}}=220 \mathrm{~kg} / \mathrm{mol}$, and the polydispersity index was PDI $=1.55$, as obtained from gel permeation chromatography. See Scheme S1 for the synthetic route.

4.4. Polymer Template. Polymer films were prepared from 10 wt \% PNIPAAm terpolymer solution in ethanol. The solution was spin-coated at room temperature onto the prefunctionalized round microscope glass substrates at 2500 rpm for $30 \mathrm{~s}$. After spin-coating, the samples were solventvapor-annealed for $1 \mathrm{~h}$ in ethanol vapor and afterward temperature-annealed for $1 \mathrm{~h}$ at $170{ }^{\circ} \mathrm{C}$ in vacuum. Then, the samples were dried for $24 \mathrm{~h}$ at $50{ }^{\circ} \mathrm{C}$ under vacuum. The polymer was cross-linked and anchored to the glass substrate by irradiation with UV light $(365 \mathrm{~nm})$ using an LED (LCS0365-07-22 High-Power LED Collimator Source, 365 nm, 7 W, $22 \mathrm{~mm}$ aperture, type B). For micropatterning, different photolithography masks with appropriate structure were positioned onto the samples.

The total irradiation energy dose for cross-linking was $4.2 \mathrm{~J} /$ $\mathrm{cm}^{2}, 0.28 \mathrm{~J} / \mathrm{cm}^{2}$ before and $3.92 \mathrm{~J} / \mathrm{cm}^{2}$ after adding the photolithography mask. These values are estimated based on a 
calibration experiment in which the intensity of UV light versus the LED current was measured without any photolithography mask between the LED and optical power meter. Thus, the real energy dose reaching the patterned PNIPAAm films can be lower. Subsequently, the samples were rinsed with pure ethanol and dried at $50{ }^{\circ} \mathrm{C}$ for $1 \mathrm{~h}$ under vacuum.

4.5. Giant Unilamellar Anchored Vesicle (GUAV) Formation. Phospholipids were purchased from SigmaAldrich as powder. 1,2-Dioleoyl-sn-glycero-3-phosphocholine (DOPC) and 1,2-dioleoyl-sn-glycero-3-phosphoserine DOPS were dissolved in chloroform $(c=1 \mathrm{~g} / \mathrm{L})$. As a fluorescent marker, Atto488-labeled 1,2-dioleoyl-sn-glycero-3-phosphoethanolamine (Atto488-DOPE) was used and dissolved in dichloromethane and methanol $(8: 2)(c=1 \mathrm{~g} / \mathrm{L})$.

Lipid solutions were stored at $-20{ }^{\circ} \mathrm{C}$ before use. The final lipid solution for GUAV formation was prepared from the DOPC solution with 20\% DOPS and 0.5\% Atto488-DOPE. The lipid solution $(30 \mu \mathrm{L})$ was added to the polymer sample. After evaporation of chloroform, $600 \mu \mathrm{L}$ PBS (phosphate buffered saline, $\mathrm{pH}$ 7.4) was used to grow GUAVs.

4.6. Confocal Laser Scanning Microscopy. Confocal laser scanning microscopy (CLSM) experiments were performed with a commercial setup (Carl Zeiss, Jena, Germany), consisting of an inverted microscope model Axiovert 200, the module LSM510, and a Zeiss C-Apochromat $40 \times / 1.2 \mathrm{~W}$ water immersion objective. The Atto488-labeled lipids were excited with an argon ion laser with a wavelength of $488 \mathrm{~nm}$. The samples were measured in Attofluor cell chambers at room temperature.

4.7. Determination of the Lipid Diffusion Coefficient via Fluorescence Correlation Spectroscopy. To the mixture of phospholipids for the GUAV formation, $0.01 \%$ Atto633-labeled DOPE was added, and the GUAVs were prepared as described above. FCS experiments were performed on an LSM 880 (Carl Zeiss, Jena, Germany) setup. Excitation laser light was focused on the samples using a Zeiss CApochromat $40 \times / 1.2 \mathrm{~W}$ water immersion objective. The emission was collected with the same objective and, after passing through a confocal pinhole, directed to a spectral detection unit (Quasar, Carl Zeiss). In this, unit emission is spectrally separated by a grating element on a 32-channel array of $\mathrm{GaAsP}$ detectors operating in a single-photon counting mode.

An argon laser $(\lambda=488 \mathrm{~nm})$ was used to excite the fluorescence of the Atto488-DOPE lipids present in the GUAVs membrane at the $0.5 \%$ fraction and thus image the vesicles and position the confocal volume precisely at the lipid membrane. Next, a HeNe laser $(\lambda=633 \mathrm{~nm})$ was used for excitation of Atto633-labeled lipids, and emission in the range from 650 to $696 \mathrm{~nm}$ was detected with a QUASAR detection unit. An Attofluor metal chamber was used as a sample cell. For each sample, 10 measurements (30 s each) were performed. Obtained experimental autocorrelation curves were fitted with a theoretical model function for a $2 \mathrm{D}$ diffusion, yielding the diffusion coefficient of the Atto633labeled lipids in the bilayer. Calibration of the size of the confocal volume was done by recording autocorrelation curves for a reference dye with a known diffusion coefficient, that is, Atto633 freely diffusing in water.

4.8. Photo-Oxidation. Erythrosine was purchased from Sigma-Aldrich as a powder and was diluted in phosphate buffered saline (PBS, $\mathrm{pH} 7.4$ ) to a concentration of $50 \mu \mathrm{M}$. For imaging, we used an LSM 880 confocal laser scanning microscope (Carl Zeiss, Jena, Germany) equipped with a Zeiss C-Apochromat $40 \times / 1.2 \mathrm{~W}$ water immersion objective. Atto488-labeled DOPE lipids were excited by an argon ion laser with a wavelength of $488 \mathrm{~nm}$, and their emission in the range from 500 to $550 \mathrm{~nm}$ was detected with the QUASAR detection unit. For irradiation of the photo-oxidizer erythrosine, we used the microscope-attached mercury lamp (HXP $120 \mathrm{C}$, FSet43wf). Image analysis was performed with the software ImageJ.

\section{ASSOCIATED CONTENT}

\section{S Supporting Information}

The Supporting Information is available free of charge on the ACS Publications website at DOI: 10.1021/acsomega.9b00912.

Swelling ratios and polymer fractions of the PNIPAAm hydrogel versus illumination energy dose; CLSM images of GUVs, formed on homogenous PNIPAAm hydrogel films; scheme of the PNIPAAm terpolymer synthetic route $(\mathrm{PDF})$

\section{AUTHOR INFORMATION}

\section{Corresponding Authors}

*E-mail: a.vagias@rug.nl (A.V.).

*E-mail: koynov@mpip-mainz.mpg.de (K.K.).

*E-mail: marques@unistra.fr (C.M.M.).

\section{ORCID ${ }^{\circ}$}

Lijun Ye: 0000-0002-9760-9263

Kaloian Koynov: 0000-0002-4062-8834

Carlos M. Marques: 0000-0002-3952-0498

Hans-Jürgen Butt: 0000-0001-5391-2618

\section{Funding}

This work was financially supported by the DFG (no. SFB 1066, Q2) and HFSP (no. RGP0013/2015).

Notes

The authors declare no competing financial interest.

\section{ACKNOWLEDGMENTS}

We thank Prof. Ulrich Jonas for the helpful discussions and Gunnar Kircher for his support with the chemical synthesis. We acknowledge the financial support by the DFG (no. SFB 1066, Q2) and HFSP (no. RGP0013/2015). C.M.M. acknowledges Max-Planck-Institut für Polymerforschung for their hospitality.

\section{ABBREVIATIONS}

DOPC: 1,2-dioleoyl-sn-glycero-3-phosphocholine; DOPE: 1,2dioleoyl-sn-glycero-3-phosphoethanolamine; DOPS: 1,2-dioleoyl-sn-glycero-3-phosphoserine; CLSM: confocal laser scanning microscopy; FCS: fluorescence correlation spectroscopy; GU(A)V: giant unilamellar (anchored) vesicle

\section{REFERENCES}

(1) Alberts, B.; Johnson, A.; Lewis, J.; Margan, D.; Raff, M.; Roberts, K.; Walter, P. Molecular Biology of the Cell; 6th ed.; Alberts, B., Johnson, A., Lewis, J., Margan, D., Raff, M., Roberts, K., Walter, P., Eds.; Garland Science: New York, Abington, 2014.

(2) Van Meer, G.; Voelker, D. R.; Feigenson, G. W. Membrane Lipids: Where They Are and How They Behave. Nat. Rev. Mol. Cell Biol. 2008, 9, 112-124.

(3) Fenz, S. F.; Sengupta, K. Giant Vesicles as Cell Models. Integr. Biol. 2012, 4, 982-995. 
(4) Walde, P.; Cosentino, K.; Engel, H.; Stano, P. Giant Vesicles: Preparations and Applications. ChemBioChem 2010, 11, 848-865.

(5) Dimova, R. Recent Developments in the Field of Bending Rigidity Measurements on Membranes. Adv. Colloid Interface Sci. 2014, 208, 225-234.

(6) Chaudhary, H.; Subramaniam, V.; Claessens, M. M. A. E. Direct Visualization of Model Membrane Remodeling by $\alpha$-Synuclein Fibrillization. ChemPhysChem 2017, 18, 1620-1626.

(7) Urakami, N.; Jimbo, T.; Sakuma, Y.; Imai, M. Molecular Mechanism of Vesicle Division Induced by Coupling between Lipid Geometry and Membrane Curvatures. Soft Matter 2018, 14, 30183027.

(8) Kamiya, K.; Takeuchi, S. Giant Liposome Formation toward the Synthesis of Well-Defined Artificial Cells. J. Mater. Chem. B 2017, 5, 5911-5923.

(9) Taylor, P.; Xu, C.; Fletcher, P. D. I.; Paunov, V. N. A Novel Technique for Preparation of Monodisperse Giant Liposomes. Chem. Commun. 2003, 1732-1733.

(10) López Mora, N.; Hansen, J. S.; Gao, Y.; Ronald, A. A.; Kieltyka, R.; Malmstadt, N.; Kros, A. Preparation of Size Tunable Giant Vesicles from Cross-Linked Dextran(Ethylene Glycol) Hydrogels. Chem. Commun. 2014, 50, 1953-1955.

(11) Le Berre, M.; Yamada, A.; Reck, L.; Chen, Y.; Baigl, D. Electroformation of Giant Phospholipid Vesicles on a Silicon Substrate: Advantages of Controllable Surface Properties. Langmuir 2008, 24, 2643-2649.

(12) Liu, X.; Zhao, R.; Zhang, Y.; Jiang, X.; Yue, J.; Jiang, P.; Zhang, Z. Using Giant Unilamellar Lipid Vesicle Micro-Patterns as Ultrasmall Reaction Containers to Observe Reversible ATP Synthesis/Hydrolysis of F0F1-ATPase Directly. Biochim. Biophys. Acta, Gen. Subj. 2007, 1770, 1620-1626.

(13) Kang, Y. J.; Wostein, H. S.; Majd, S. A Simple and Versatile Method for the Formation of Arrays of Giant Vesicles with Controlled Size and Composition. Adv. Mater. 2013, 25, 6834-6838.

(14) Osaki, T.; Kamiya, K.; Kawano, R.; Iino, R.; Noji, H.; Takeuchi, $\mathrm{S}$. Uniform-Sized Proteoliposome Formation by Using Electrospray for Microscopic Membrane Protein Assays. In Proceedings of 17th International Conference on Miniaturized Systems for Chemistry and Life Sciences; 2013.

(15) Zhu, C.; Li, Q.; Dong, M.; Han, X. Giant Unilamellar Vesicle Microarrays for Cell Function Study. Anal. Chem. 2018, 90, 1436314367.

(16) Gertrude Gutierrez, M.; Yoshida, S.; Malmstadt, N.; Takeuchi, S. Photolithographic Patterned Surface Forms Size-Controlled Lipid Vesicles. APL Bioeng. 2018, 2, No. 016104.

(17) Fan, T.; Wang, Q.; Hu, N.; Liao, Y.; Chen, X.; Wang, Z.; Yang, Z.; Yang, J.; Qian, S. Preparation of Giant Lipid Vesicles with Controllable Sizes by a Modified Hydrophilic Polydimethylsiloxane Microarray Chip. J. Colloid Interface Sci. 2019, 536, 53-61.

(18) Han, W. B.; Kwak, R.; Kang, J. Y.; Kim, T. S. Generation of Solvent-Free 3D Lipid Structure Arrays on High Aspect Ratio $\mathrm{Si}$ Microwell Substrate. Adv. Mater. Interfaces 2019, 6, 1801554.

(19) Beines, P. W.; Klosterkamp, I.; Menges, B.; Jonas, U.; Knoll, W. Responsive Thin Hydrogel Layers from Photo-Cross-Linkable Poly(N-Isopropylacrylamide) Terpolymers. Langmuir 2007, 23, 2231-2238.

(20) Giannelli, M.; Beines, P. W.; Roskamp, R. F.; Koynov, K.; Fytas, G.; Knoll, W. Local and Global Dynamics of Transient Polymer Networks and Swollen Gels Anchored on Solid Surfaces. J. Phys. Chem. C 2007, 111, 13205-13211.

(21) Raccis, R.; Roskamp, R.; Hopp, I.; Menges, B.; Koynov, K.; Jonas, U.; Knoll, W.; Butt, H. J.; Fytas, G. Probing Mobility and Structural Inhomogeneities in Grafted Hydrogel Films by Fluorescence Correlation Spectroscopy. Soft Matter 2011, 7, 7042-7053.

(22) Vagias, A.; Košovan, P.; Koynov, K.; Holm, C.; Butt, H. J.; Fytas, G. Dynamics in Stimuli-Responsive Poly(N-Isopropylacrylamide) Hydrogel Layers as Revealed by Fluorescence Correlation Spectroscopy. Macromolecules 2014, 47, 5303-5312.
(23) Fickert, J.; Makowski, M.; Kappl, M.; Landfester, K.; Crespy, D. Efficient Encapsulation of Self-Healing Agents in Polymer Nanocontainers Functionalized by Orthogonal Reactions. Macromolecules 2012, 45, 6324-6332.

(24) Weinberger, A.; Tsai, F.-C.; Koenderink, G. H.; Schmidt, T. F.; Itri, R.; Meier, W.; Schmatko, T.; Schroder, A.; Marques, C. GelAssisted Formation of Giant Unilamellar Vesicles. Biophys. J. 2013, $105,154-164$.

(25) Horger, K. S.; Estes, D. J.; Capone, R.; Mayer, M. Films of Agarose Enable Rapid Formation of Giant Liposomes in Solutions of Physiologic Ionic Strength. J. Am. Chem. Soc. 2009, 131, 1810-1819.

(26) Lira, R. B.; Steinkühler, J.; Knorr, R. L.; Dimova, R.; Riske, K. A. Posing for a Picture: Vesicle Immobilization in Agarose Gel. Sci. Rep. 2016, 6, 25254.

(27) Bagatolli, L. Chapter 10. In The Giant Vesicle Book; Dimova, R., Marques, C., Eds.; CRC press: 2019.

(28) Vagias, A.; Sergelen, K.; Koynov, K.; Košovan, P.; Dostalek, J.; Jonas, U.; Knoll, W.; Fytas, G. Diffusion and Permeation of Labeled IgG in Grafted Hydrogels. Macromolecules 2017, 50, 4770-4779.

(29) García-Sáez, A. J.; Schwille, P. Fluorescence Correlation Spectroscopy for the Study of Membrane Dynamics and Protein/ Lipid Interactions. Methods 2008, 46, 116-122.

(30) Chiantia, S.; Ries, J.; Schwille, P. Fluorescence Correlation Spectroscopy in Membrane Structure Elucidation. Biochim. Biophys. Acta, Biomembr. 2009, 1788, 225-233.

(31) Guo, L.; Har, J. Y.; Sankaran, J.; Hong, Y.; Kannan, B.; Wohland, T. Molecular Diffusion Measurement in Lipid Bilayers over Wide Concentration Ranges: A Comparative Study. ChemPhysChem 2008, 9, 721-728.

(32) Kriegsmann, J.; Gregor, I.; Von Der Hocht, I.; Klare, J.; Engelhard, M.; Enderlein, J.; Fitter, J. Translational Diffusion and Interaction of a Photoreceptor and Its Cognate Transducer Observed in Giant Unilamellar Vesicles by Using Dual-Focus FCS. ChemBioChem 2009, 10, 1823-1829.

(33) Thomas, F. A.; Visco, I.; Petrášek, Z.; Heinemann, F.; Schwille, P. Diffusion Coefficients and Dissociation Constants of Enhanced Green Fluorescent Protein Binding to Free Standing Membranes. Data Brief 2015, 5, 537-541.

(34) Stratton, S. P.; Liebler, D. C. Determination of Singlet OxygenSpecific versus Radical-Mediated Lipid Peroxidation in Photosensitized Oxidation of Lipid Bilayers: Effect of $\beta$-Carotene and $\alpha$ Tocopherol. Biochemistry 1997, 36, 12911-12920.

(35) Caetano, W.; Haddad, P. S.; Itri, R.; Severino, D.; Vieira, V. C.; Baptista, M. S.; Schröder, A. P.; Marques, C. M. Photo-Induced Destruction of Giant Vesicles in Methylene Blue Solutions. Langmuir 2007, 23, 1307-1314.

(36) Banerjee, C.; Westberg, M.; Breitenbach, T.; Bregnhøj, M.; Ogilby, P. R. Monitoring Interfacial Lipid Oxidation in Oil-in-Water Emulsions Using Spatially Resolved Optical Techniques. Anal. Chem. 2017, 89, 6239-6247.

(37) Riske, K. A.; Sudbrack, T. P.; Archilha, N. L.; Uchoa, A. F.; Schroder, A. P.; Marques, C. M.; Baptista, M. S.; Itri, R. Giant Vesicles under Oxidative Stress Induced by a Membrane-Anchored Photosensitizer. Biophys. J. 2009, 97, 1362-1370.

(38) Weber, G.; Charitat, T.; Baptista, M. S.; Uchoa, A. F.; Pavani, C.; Junqueira, H. C.; Guo, Y.; Baulin, V. A.; Itri, R.; Marques, C. M.; et al. Lipid Oxidation Induces Structural Changes in Biomimetic Membranes. Soft Matter 2014, 10, 4241.

(39) Guo, Y.; Baulin, V. A.; Thalmann, F. Peroxidised Phospholipid Bilayers: Insight from Coarse-Grained Molecular Dynamics Simulations. Soft Matter 2016, 12, 263-271. 